\title{
Dust from Reionization
}

\author{
Erik Elfgren ${ }^{1}$ and François-Xavier Désert ${ }^{2}$
}

1 Department of Physics, Luleå University of Technology, SE-971 87 Luleå, Sweden

${ }^{2}$ Laboratoire d'Astrophysique, Observatoire de Grenoble, BP 53, 414 rue de la piscine, 38041 Grenoble Cedex 9, France

Received $;$ date $_{i} /$ Accepted $;$ date $_{i}$

\begin{abstract}
The possibility that population III stars have reionized the Universe at redshifts greater than 6 has recently gained momentum with WMAP polarization results. Here we analyse the role of early dust produced by these stars and ejected into the intergalactic medium. We show that this dust, heated by the radiation from the same population III stars, produces a submillimetre excess. The electromagnetic spectrum of this excess could account for a significant fraction of the FIRAS (Far Infrared Absolute Spectrophotometer) cosmic far infrared background above 700 micron. This spectrum, a primary anisotropy $(\Delta T)$ spectrum times the $v^{2}$ dust emissivity law, peaking in the submillimetre domain around 750 micron, is generic and does not depend on other detailed dust properties. Arcminute-scale anisotropies, coming from inhomogeneities in this early dust, could be detected by future submillimetre experiments such as Planck HFI.
\end{abstract}

Key words. Dust - CMB - Reionization

\section{Introduction}

More accurate measurements of the cosmic microwave background $(\mathrm{CMB})$ implies a need for a better understanding of the different foregrounds. We study the impact of dust in the very early universe $5<z<15$. WMAP data on the CMB polarization, Kogut et al. (2003) provides a strong evidence for a rather large Thomson opacity during the reionization of the Universe: $\tau_{e}=0.17 \pm 0.04$ (68\%C.L.). Although the mechanism of producing such an opacity is not fully understood, Cen, R. $(2002,2003)$ has shown that early, massive population-III (Pop III) stars could ionize the Universe within $5<z<15$ (see Fig. 1 and Fig. 2). Adopting this hypothesis, we discuss the role and observability of the dust that is produced by the Pop III stars. As we can only conjecture about the physical properties and the abundance of this early dust, we adopt a simple dust grain model with parameters deduced from the Milky Way situation. The dust production is simply linked to the ionizing photon production by the stars through their thermal nuclear reactions. The low potential well of the small pre-galactic halos allows the ejected dust to be widely spread in the intergalactic medium. The ionizing and visible photons from the same Pop III stars heat this dust. There are no direct measurements of this dust, but by means of other results the amount of dust can be estimated. A similar study has been done for a later epoch of the universe, in which data are more readily available, Pei et al. (1999). We use a cosmology with $\Omega_{t o t}=\Omega_{m}+\Omega_{\Lambda}=1$, where $\Omega_{m}=\Omega_{b}+\Omega_{D M}=0.133 / h^{2}, \Omega_{b}=0.0226 / h^{2}$ and $h=0.72$ as advocated by WMAP, Spergel et al. (2003), using WMAP data in combination with large scale structure ob-

Send offprint requests to: Erik Elfgren, e-mail: elf@ludd.luth. se

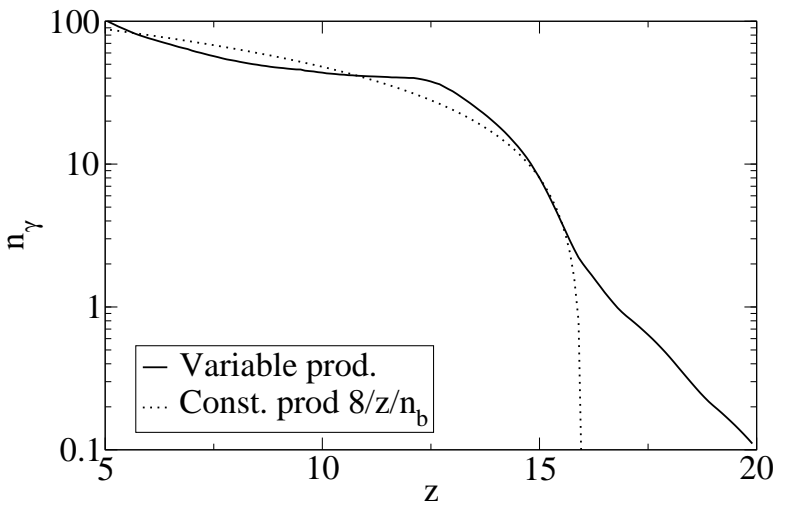

Fig. 1. Total number of ionizing photons produced from Pop III stars per baryon, cf. (Cen, R. 2002, figure 14). The dotted line represents a simplified model with a constant photon production, from $z=16$, of 8 per unit $z$ per baryon. The results are similar.

servations (2dFGRS + Lyman $\alpha$ ). Furthermore, since $z \gg 1$ the universe is matter-dominated. We relate all cosmological parameters to their measurement today so that they have their present-day values throughout our calculations.

We now proceed to compute the abundance and the temperature of this dust. Consequences on the CMB distortions are then to be discussed. 


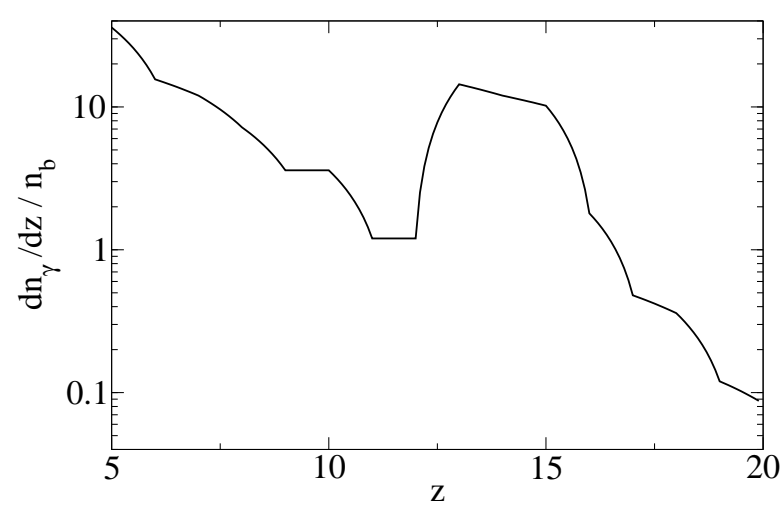

Fig. 2. Production rate of ionizing photons from Pop III stars per baryon, $\frac{d n_{\gamma}}{d z} / n_{b}$. The odd form between each integer $z$ is not physical but are due to the fact that the redshift $z$ is a nonlinear function of time.

\section{Dust Model}

Here we assume the dust properties to be similar to what is observed in our galaxy. For simplicity, we suppose spherical dust grains with radius $a=0.1 \mu \mathrm{m}$ and density $\rho_{g}=2.3 \cdot 10^{3}$ $\mathrm{kg} / \mathrm{m}^{3}$. The absorption cross section, $\sigma_{v}$, between photons and dust can be written as

$\sigma_{v}=Q_{v} \pi a^{2}$,

where we parametrize the frequency dependency as

$Q_{v}= \begin{cases}Q_{0} \frac{a}{a_{r}}\left(\frac{v}{v_{r}}\right)^{\beta_{v}} & \text { submm and infra red (IR), } \\ 1 & \text { visible and ultra violet (UV), }\end{cases}$

where $v_{r}, a_{r}$ and $Q_{0}$ are normalization constants. There is only one independent constant which means that we can fix $a_{r}=$ $0.1 \mu \mathrm{m}$. In (Desert et al. 1990, figure 3) the poorly known knee wavelength, $\lambda_{r}=c / v_{r}$ was set to $100 \mu \mathrm{m}$. Here, we choose $40 \mu \mathrm{m}$ for simplicity, so that early dust radiates mostly in the $v^{2}$ emissivity regime. Above the characteristic frequency $v_{r}$ the spectral index $\beta=1$, below $\beta=2$. The exact position of $v_{r}$ is not very important for our study because it is mainly above the interesting wave-length region $\sim 0.3-3 \mathrm{~mm}$ and it will not change the magnitude of the signal.

In the submm and far IR range, the spectral index is constant, and with $Q_{0}=0.0088$ the assumed opacity agrees well with measurements by FIRAS on cirrus clouds in our galaxy, cf. Boulanger et al. (1996); Fixsen et al. (1998); Lagache et al. (1999). In the visible and UV region, the cross section is independent of the frequency because $\lambda<2 \pi a$. In the submm region, the cross section is proportional to the mass of the grain.

In order to evaluate the significance of the dust during the reionization, we calculate the amount of dust present in the universe at a given time. The co-moving relative dust density is $\Omega_{d, 0}=\rho_{d}(z) /\left((1+z)^{3} \rho_{c}\right)$, where $\rho_{d}(z)$ is the dust density, $z$ is the red-shift, $\rho_{c}=\frac{3 H_{0}^{2}}{8 \pi G}$ is the critical density $\left(H_{0}\right.$ and $G$ are Hubble's and Newton's constants, respectively). The comoving relative dust density as measured today evolves as:

$\frac{d \Omega_{d, 0}}{d z}=J_{+}-J_{-}$,

where $J_{+}$and $J_{-}$are the production and the destruction rate respectively.

The Pop III stars produce enough photons for the reionization while burning $H$ and thus forming metals ( $\mathrm{Li}$ and higher). These metals are released in supernovae explosions at the end of the stars short lives ( $\sim 1 \mathrm{Myr})$, whereafter they clump together to form dust, Nozawa et al. (2003). Knowing the production rate of ionizing photons to be $\frac{d n_{\gamma}}{d z} / n_{b}$ (Fig. 2), we can calculate the total photon energy released from the Pop III stars. This can be done by supposing that each photon has an effective energy of $E_{\gamma}=c_{\gamma} \int_{v_{\text {ion }}}^{\infty} d v h v B_{v}\left(T_{*}\right) / \int_{v_{\text {ion }}}^{\infty} d v B_{v}\left(T_{*}\right)$, where $h v_{\text {ion }}=13.6 \mathrm{eV}$ and $B_{v}\left(T_{*}\right)$ is the spectrum of a star with temperature $T_{*}$. The energy of the non-ionizing photons is included through $c_{\gamma}=u_{t o t} / u_{v>v_{\text {ion }}}$ ( $u$ is the energy from the star). A Pop III star has $T_{*} \sim 80000 \mathrm{~K}$ (Shioya et al. 2002, page 9) which gives $E_{\gamma} \approx 36 \mathrm{eV}$. Note that for other reasonable star temperatures, $E_{\gamma}$ does not vary significantly, $\left.E_{\gamma}\right|_{60 \times 10^{3} \mathrm{~K}} \approx 36 \mathrm{eV}$ and $\left.E_{\gamma}\right|_{100 \times 10^{3} K} \approx 40 \mathrm{eV}$. Hence, the total Pop III photon energy production is $E_{\gamma} \frac{d n_{\gamma}}{d z} / n_{b}$ per baryon per unit $z$. For each consumed nucleon, we assume that a nuclear energy of $E_{r}=7$ $\mathrm{MeV}$ is released as radiation, which means that the nucleon consumption rate is $\frac{E_{\gamma}}{E_{r}} \frac{d n_{\gamma}}{d z} / n_{b}$ nucleons per baryon per unit $z$. If $f_{d}$ is the fraction of the consumed baryon mass that becomes interstellar dust, (some of the metal atoms will remain with the core after the $\mathrm{SN}$ explosion, some will stay in the close vicinity of the SN and some will never clump together to form dust) the co-moving dust production rate will be

$J_{+}=f_{d} \frac{E_{\gamma}}{E_{r}} \Omega_{b} \frac{d n_{\gamma}}{d z} / n_{b}$.

A dust grain will eventually be destroyed, e.g. by collision, by supernova shockwaves or by cosmic rays, see Draine $\&$ Salpeter (1979) for further discussion. If a dust grain has a lifetime of $\Delta t$ we can write the dust destruction rate as

$J_{-}=\frac{\Omega_{d, 0}(z)}{\Delta t} \frac{d t}{d z} \approx-\frac{\Omega_{d, 0}(z)}{\Delta t H_{0} \Omega_{m}^{1 / 2}(1+z)^{5 / 2}}$,

where $\Omega_{m}$ is the relative matter content today, because the universe is matter dominated for $5<z<15$.

Solving Eq. 3 gives the dust density evolution

$\Omega_{d, 0}(z)=\int_{z}^{z_{i}} J_{+}\left(z^{\prime}\right) \frac{Y\left(z^{\prime}\right)}{Y(z)} d z^{\prime}$,

where $z_{i}=20$ is the beginning of the dust formation (see Fig. 1) and

$Y(z)=\exp \left(\frac{2}{3} \frac{(1+z)^{-3 / 2}}{\Omega_{m}^{1 / 2} \Delta t H_{0}}\right)$.

We note that the source term $J_{+}$is modulated by the destruction term $\frac{Y\left(z^{\prime}\right)}{Y(z)}$. The dust density is plotted in Fig. 3 where we note a strong dependency on the dust lifetime. In local dust $\Delta t \sim$ 100 Myr, Draine \& Salpeter (1979). However, the uncertainty 


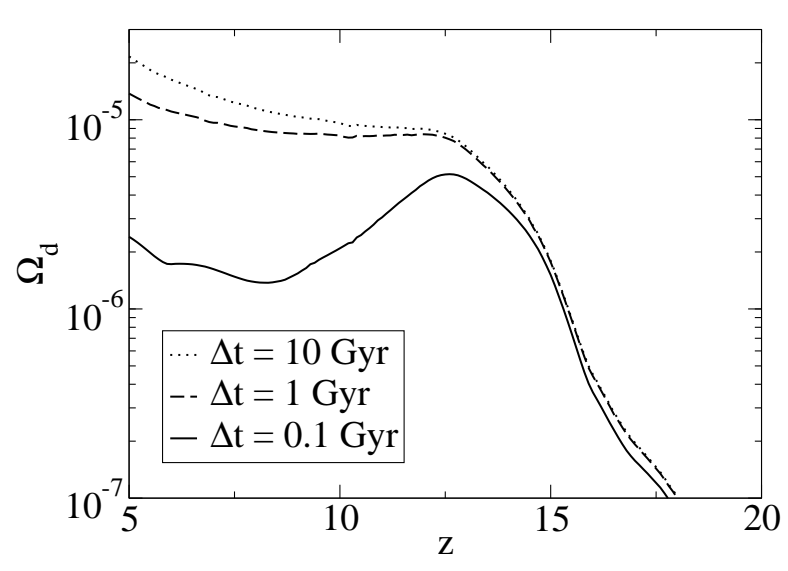

Fig. 3. The co-moving relative dust density evolution $\Omega_{d, 0}=$ $\rho_{\text {dust }} / \rho_{c}$, for $f_{d}=1$. The minima at $z=6$ and 9 for $\Delta t \leq 0.1$ Gyr is due to the fact that $\Delta z=1$ is not a constant time interval.

is rather large, according to Draine (1990), $\Delta t=30 \mathrm{Myr}-10$ Gyr, depending on the environment. Note, however, that the density at the reionization red-shifts is much lower than in the interstellar medium in the Milky Way which implies a rather long dust life-time.

\section{Results and Discussion}

\subsection{Metallicity}

If we suppose that most of the metals were ejected as dust (not as gas) the metallicity comes from the dust grains. The metallicity is directly obtained through the produced dust. By letting $\Delta t \rightarrow \infty(\Delta t=10 \mathrm{Gyr}$ is good enough $)$ we find the metallicity:

$\frac{Z}{Z_{\odot}}=\frac{\Omega_{d, 0}(\Delta t \rightarrow \infty)}{0.02 \cdot \Omega_{b}} \approx 1147 \cdot \Omega_{d, 0}(\Delta t \rightarrow \infty)$

or in absolute terms $Z \approx 22.9 \Omega_{d, 0}$. At $z=5$ we have $\Omega_{d, 0}=$ $2.3 \cdot 10^{-5} f_{d}$, which gives $Z \approx 5.2 \cdot 10^{-4} f_{d}=0.026 f_{d} Z_{\odot}$.

There are not much metallicity data available for $z>5$. Metal poor stars in our galaxy are one point of reference, absorption lines in the Ly $\alpha$ spectrum from quasars are another one. The lowest metallicities found in stars in the Milky Way are $Z / Z_{\odot} \sim 0.01$, Depagne et al. (2002). The Ly $\alpha$ forest suggests (Songaila \& Cowie 2002, figure 13) that $Z / Z_{\odot} \sim 0.003$ for $z \sim 4.5$ assuming that $[\mathrm{Fe} / \mathrm{H}] \approx \log \left(Z / Z_{\odot}\right)$ as suggested by (VandenBerg et al. 2000, page 432). This indicates that $f_{d} \sim 0.1$. However, this might be lower than the actual value, cf. (Pettini et al. 1997, figure 4).

In heavy stars, virtually all the helium is consumed, producing metals. For simplicity (and lack of data), we assume that all the ejected metals clump to form dust, $f_{d} \approx f_{\text {eject }}$. This means that $f_{d}$ will almost entirely depend on the dust ejection rate in the supernova explosion. In Iwamoto et al. (1998) a detected hypernova of mass $M \sim 14 M_{\odot}$ seems to have $f_{\text {eject }} \gtrsim 0.7$. Furthermore, according to a dust production model by Nozawa et al. (2003), $f_{d} \approx 0.2-0.3$. At the same time, some of the stars will become black holes, not ejecting any metals, Heger $\&$ Woosley (2002), decreasing $f_{d}$. Currently this decrease is largely unknown.

In summary, the mass fraction of the produced metals in the Pop III stars, having become interstellar dust, should be around $f_{d} \sim 0.1-0.3$. In the following we use the more conservative $f_{d}=0.1$, in agreement with the Ly $\alpha$ forest measurements, unless otherwise stated.

\subsection{Dust Opacity}

With our model for the dust density evolution, we want to calculate the opacity of the dust, as seen by the CMB. This will tell us how much the CMB spectrum is altered by the passage through the dust.

The dust opacity is given by

$$
\begin{aligned}
\tau_{v} & =c \int d z \frac{d t}{d z} \sigma_{v_{e}} n_{d}(z) \\
& =\frac{Q_{0} c}{\sqrt{\Omega_{m}} a_{r} H_{0}} \frac{3}{4} \frac{\rho_{c}}{\rho_{g}} \int d z\left(\frac{v}{v_{r}}\right)^{\beta_{v_{e}}} \Omega_{d, 0}(z)(1+z)^{1 / 2+\beta_{v_{e}}},
\end{aligned}
$$

where $v\left(v_{e}\right)$ is the observed (emitted) frequency and $v=$ $v_{e} /(1+z)$. The dust number density is $n_{d}(z)=(1+z)^{3} \times$ $\rho_{c} \Omega_{d, 0}(z) / m_{g}$ where $m_{g}=\frac{4 \pi a^{3}}{3} \rho_{g}$ is the grain mass. We see (from $\tau \propto \Omega_{d, 0}$ ) that $\tau$ is proportional to the parameter $f_{d}$.

The resulting opacity can be seen in Fig. 4. We note that the opacity is small, $\tau \ll 1$. The smooth knee is due to the change of $\beta$ at the redshifted $v_{r}$, see Sect. 2, but this is not in the spectral range of the CMB. The differential opacity $d \tau / d z$ is plotted in Fig. 5 for $\lambda=1 \mathrm{~mm}$. We see that with a short dust lifetime, the dust differential opacity falls off almost immediately (in terms of $z$ ). However, for longer lifetimes, the early dust could still play a certain role for $z<3$. This could eventually contribute to dimming of distant objects. We also note the impact of the expansion of the universe in decreasing the dust density and thus the opacity. This is why the increase in Fig. 1, at $z \sim 5$, is not apparent in the opacity, Fig. 4. Furthermore, the submillimetre effective dust opacity follows a $v^{2}$ emissivity law.

\subsection{Dust Temperature}

In order to deduce the equilibrium temperature of the dust, we write the balance between the absorbed CMB, the absorbed starlight and the emitted IR light from the dust:

$P_{d}=P_{*}+P_{C M B}$.

The powers $P_{d}$ and $P_{C M B}$ can be written as

$P_{X}=4 \pi \int_{0}^{\infty} d v_{e} \sigma_{v_{e}} B_{v_{e}}\left(T_{X}\right)$

where $B_{v}$ is a Planck blackbody spectrum and $X=\{C M B, d\}$. In the wave-length range considered, the spectral index $\beta=2$. Supposing that $\beta$ is constant, eq. 11 can be solved for the dust temperature analytically in the submm range:

$T_{d}^{4+\beta}=T_{* e f f}^{4+\beta}+T_{C M B}^{4+\beta}$, 


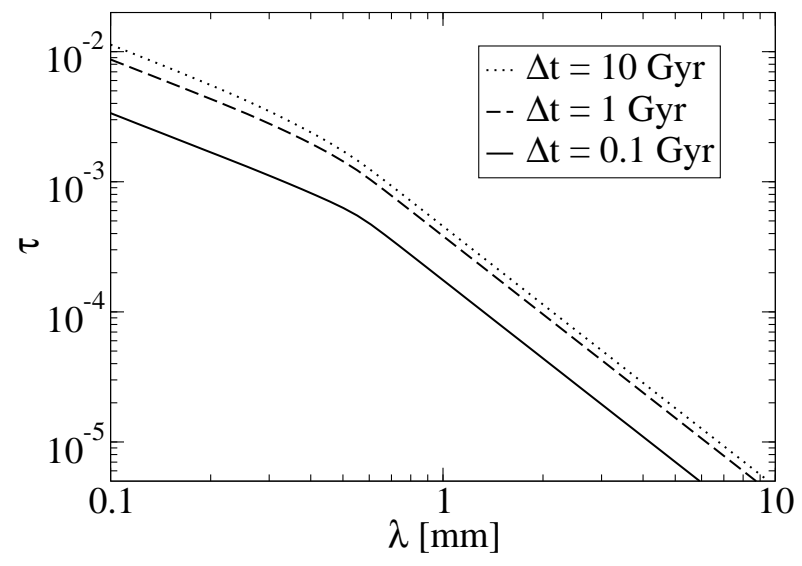

Fig. 4. Opacity $\tau$ with dust evolution taken into account.

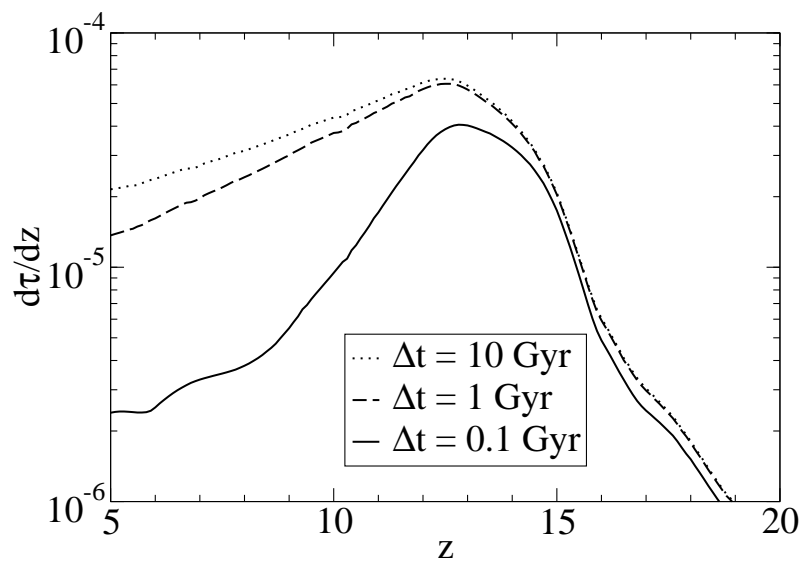

Fig. 5. The differential opacity $d \tau / d z$ at $\lambda=1 \mathrm{~mm}$ for different dust lifetimes.

where the effective temperature is defined by

$T_{* e f f}^{4+\beta}=\frac{P_{*}}{8 \pi^{2} h c^{-2}\left(Q_{0} \cdot\left(a^{3} / a_{r}\right) v_{r}^{-\beta}\right)\left(k_{B} / h\right)^{4+\beta} C_{\beta}}$

and $C_{\beta}=\int_{0}^{\infty} d x x^{3+\beta} /\left(e^{x}-1\right)=(\beta+3) ! \sum_{k=1}^{\infty} k^{-(4+\beta)}$, such that $C_{0} \approx 6.494, C_{1} \approx 24.89$ and $C_{2} \approx 122.1$.

However, in our calculations we use the exact eq. 11 and 12 , while eq. 14 can be used as a cross-check.

The absorbed power density, $P_{*}$ from the radiation of Pop III stars peaks in the UV-region and can be approximated by

$P_{*}=\sigma_{U V} u_{*}(z) c$,

where $\sigma_{U V}$ is the dust-photon cross section in the UV region and the energy density is

$u_{*}(z)=f_{\text {esc }} \int_{z_{i}}^{z} d z^{\prime} \frac{d n_{\gamma}}{d z^{\prime}} E_{\gamma}\left(\frac{1+z}{1+z^{\prime}}\right)^{4}$,

where $f_{\text {esc }}$ is the escape fraction of photons from the star halos. We neglect the loss of photons due to the reionization itself.

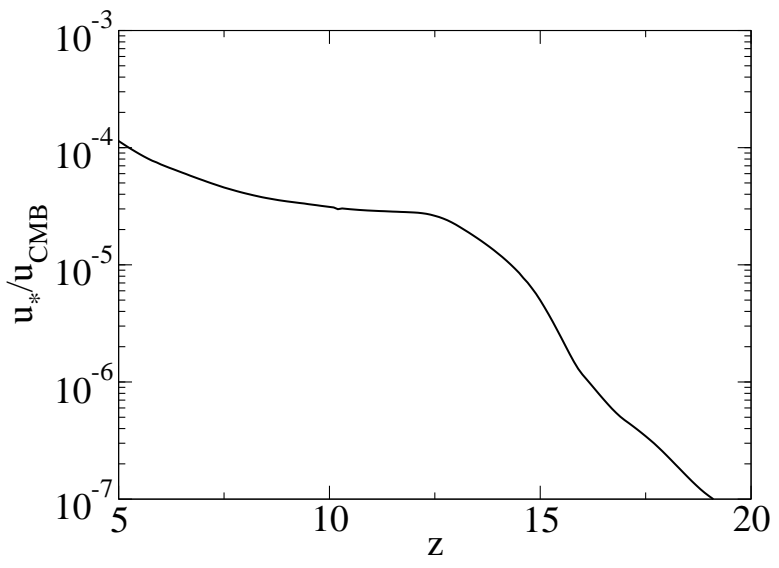

Fig. 6. Energy density of ionizing photons compared to $u_{C M B}=$ $4 \sigma_{S} T_{C M B}^{4} / c$, where $\sigma_{S}$ is Stefan-Boltzmann's constant.

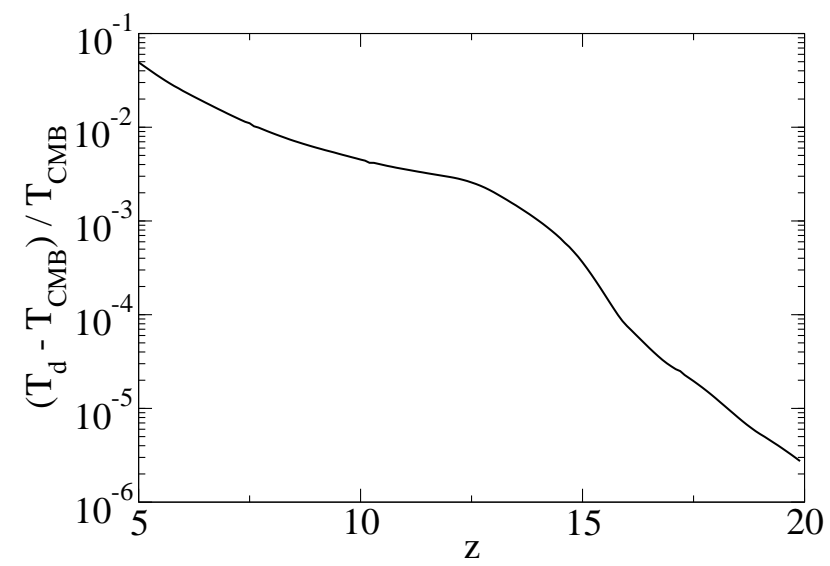

Fig. 7. The dust temperature is plotted against the CMB temperature with the relative quatity $\left(T_{d}-T_{C M B}\right) / T_{C M B}$.

$E_{\gamma} \frac{1+z}{1+z^{\prime}}$ is the effective energy of the photon emitted at $z^{\prime}$ and then redshifted to $z$. According to Cen, R. (2003), $f_{\text {esc }}=0.3$ gives an electron opacity $\tau_{e} \approx 0.13$ which is within one standard deviation of the results by WMAP. Hereafter, we adopt this value of $f_{\text {esc }}$.

The energy density of the ionizing photons are compared to the CMB in Fig. 6. The star energy density is much less than the CMB energy density at this epoch, and the curve resembles the accumulated photons in Fig. 1. Hence, the dust temperature closely follows the CMB temperature, see Fig. 7 and Eq. 12.

\subsection{Observed Intensity}

Now we proceed to compute the average intensity (monopole term) of the submm and microwave background which is made of the CMB and early dust emission. The simple radiative 


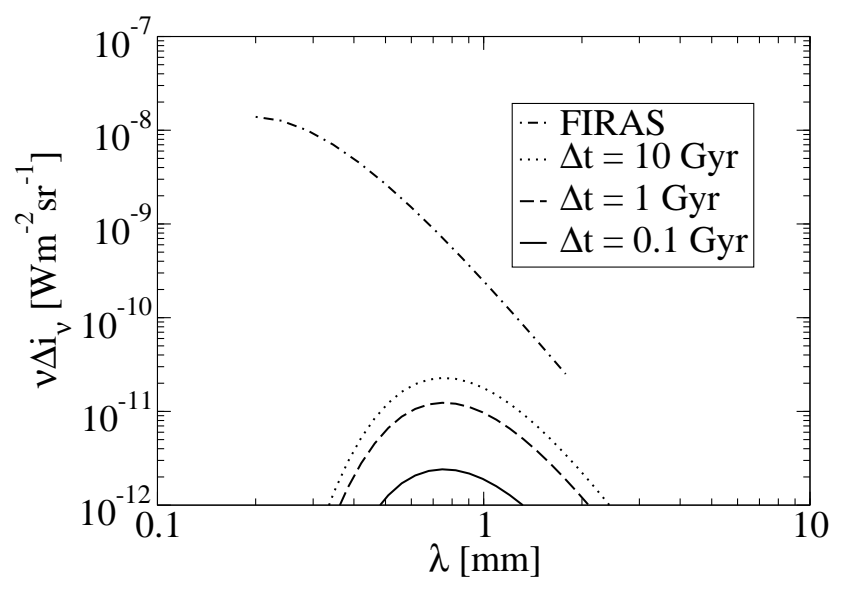

Fig. 8. Comparison of the modeled intensity for the early dust emission in excess of the CMB with the observed FIRAS spectrum (dashed red curve) of the cosmic far IR background as detailed by Lagache et al. (1999).

transfer of the CMB through the uniform dust screen yields the following observed intensity:

$i_{v}=e^{-\tau_{v}}\left[B_{v}\left(T_{C M B}\right)+\int_{0}^{\tau_{v}} e^{\tau_{e}} \frac{B_{v_{e}}\left(T_{d}(z)\right)}{(1+z)^{3}} d \tau_{e}\right]$.

From Fig. 4 and 7, we see that the opacity is small, $(\tau \ll 1)$ and the dust temperature is only slightly higher than the CMB temperature $\left(T_{d} \gtrsim T_{C M B}\right)$. This gives the following formula for the excess intensity relative to the unperturbed CMB:

$$
\begin{aligned}
\Delta i_{v} & \equiv i_{v}-B_{v}\left(T_{C M B}\right) \\
& \left.\approx T_{C M B} \frac{d B_{v}}{d T}\right|_{T=T_{C M B}} \int_{0}^{\tau_{v}} \frac{T_{d}(z)-T_{C M B}(z)}{T_{C M B}(z)} d \tau_{e},
\end{aligned}
$$

where $T_{C M B}$ is the CMB temperature today. The integrant is plotted in Fig. 7. We note that a new component is added to the primary CMB spectrum. Eq. 18 tells us that it has a specific spectrum which is the product of a $2.725 \mathrm{~K}$ blackbody temperature fluctuation spectrum (like primary anisotropies) and a $v^{2}$ power law (from $d \tau_{e}$ ). This effect is mostly visible in the submm range and has a minor contribution in the radio domain.

In Fig. 8, the excess intensity is plotted along with the extragalactic background measured by FIRAS, Puget et al. (1996); Fixsen et al. (1998); Lagache et al. (1999). Depending on the dust destruction rate (parametrized by the dust lifetime $\Delta t$ ), the computed early dust background can be an important part of the observed background from $400 \mu \mathrm{m}$ up to the mm wavelength. The exact position of $\lambda_{r}$ will only slightly displace the spectrum, leaving the magnitude unchanged. Most of the far IR background can now be explained by a population of $z=0$ to $z=3$ luminous IR galaxies, Gispert et al. (2000). A fraction of the submillimetre part of this background could arise from larger redshift dust emission as suggested by Fig. 8 .

In order to check our results, we calculate the co-moving luminosity density of the dust in the submm region and compare it with (Gispert et al. 2000, figure 4). We find them compatible.

\subsection{Discussion}

Just like the Thomson scattering during reionization, early dust will also tend to erase the primordial anisotropies in the CMB. However, due to the much smaller dust opacity (compare $\tau_{d}(1 \mathrm{~mm}) \lesssim 10^{-3}$ and $\left.\tau_{e}=0.17\right)$, this effect will be negligible.

The early dust will also introduce a new type of secondary anisotropies with a typical size of a dark matter filament. Here, we only estimate the order of magnitude of this effect. If the comoving size of the dark matter filament is $L$, the angular size is $3 \cdot(L / 5 \mathrm{Mpc})$ arcminutes at $z=10$ which corresponds to multipole number $\ell \sim 4000 \cdot(L / 5 \mathrm{Mpc})$. Fortunately, this region in $\ell$-space does not contain any primordial fluctuations because of the Silk damping. However, there are other foregrounds in the same region, see Aghanim et al. (2000). If we suppose a contrast of $10 \%$ in the dust intensity between dark matter filaments and the void, we obtain values of $\Delta T / T \approx 3 \times 10^{-7}$ (for $\lambda=1 \mathrm{~mm}, f_{d}=0.1$ and $\Delta t=1 \mathrm{Gyr}$ ). These anisotropies, pending more accurate calculations, clearly are in the range of expected arcminute secondary anisotropies from other effects. They could be detected by Planck HFI (High Frequency Instrument), Lamarre et al. (2003) and FIRAS-II type of instrument, Fixsen \& Mather (2002).

The results of these calculations depend only very weakly on the precise dust model assumptions. We have also tried a different (but similar) shape of the ionizing photon production, Fig. 1, and found that the results do not vary significantly.

Very little is known about the universe during the reionization epoch. Nevertheless, there are several parameters that could be calculated more accurately.

The two most important parameters in the present model are the dust lifetime, $\Delta t$ and the mass fraction of the produced metals that are ejected as interstellar dust, $f_{d}$. The dust lifetime could be determined more precisely by making 3D simulations of the dust production in combination with structure formation. The simulations would also give the inhomogeneous dust density evolution. The result would be a better estimate of the aforementioned secondary anisotropies caused by the variations in the dust opacity. A more refined dust grain model, using e.g. a distribution of grain sizes would also be more realistic. If the dust is long-lived, it could also have a certain impact on measurements in the optical and UV region. Finally, we note that most of the results are proportional to the dust density and thus to $f_{d}$. To evaluate $f_{d}$ more precisely, we need a better understanding of the typical properties of the first generation of stars, see section 3.1, which is currently much debated.

\section{Conclusions}

We have shown that the radiation from early dust, produced and heated by Pop III stars, contributes to the extragalactic submillimetre background within the limits set by FIRAS. It may not be detected by the present generation of instruments but future experiments such as Planck HFI and FIRAS-II should be able to measure it, by using its specific predicted spectral signature. This high-redshift dust, contemporary to the reionization, should show up as small-scale anisotropies when observed by 
sensitive submillimetre instruments. These anisotropies are in the same range as other small-scale anisotropy effects.

\section{References}

Aghanim, N., Balland, C., \& Silk, J. 2000, A\&A, 357, 1

Boulanger, F., Abergel, A., Bernard, J.-P., et al. 1996, A\&A, 312,256

Cen, R. 2002, ApJ, 591, 12

Cen, R. 2003, ApJL, 591, L5

Depagne, E., Hill, V., Spite, M., et al. 2002, A\&A, 390, 187

Desert, F.-X., Boulanger, F., \& Puget, J. L. 1990, A\&A, 237, 215

Draine, B. T. 1990, in ASP Conf. Ser. 12: The Evolution of the Interstellar Medium, 193-205

Draine, B. T. \& Salpeter, E. E. 1979, ApJ, 231, 438

Fixsen, D. J., Dwek, E., Mather, J. C., Bennett, C. L., \& Shafer, R. A. 1998, ApJ, 508, 123

Fixsen, D. J. \& Mather, J. C. 2002, ApJ, 581, 817

Gispert, R., Lagache, G., \& Puget, J. L. 2000, A\&A, 360, 1

Heger, A. \& Woosley, S. E. 2002, ApJ, 567, 532

Iwamoto, K., Mazzali, P. A., Nomoto, K., et al. 1998, Nature, 395, 672

Kogut, A., Spergel, D. N., Barnes, C., et al. 2003, ApJS, 148, 161

Lagache, G., Abergel, A., Boulanger, F., Désert, F. X., \& Puget, J.-L. 1999, A\&A, 344, 322

Lamarre, J. M., Puget, J. L., Bouchet, F., et al. 2003, ArXiv Astrophysics e-prints, astro-ph/0308075

Nozawa, T., Kozasa, T., Umeda, H., Maeda, K., \& Nomoto, K. 2003, ArXiv Astrophysics e-prints, astro-ph/0307108

Pei, Y. C., Fall, S. M., \& Hauser, M. G. 1999, ApJ, 522, 604

Pettini, M., Smith, L. J., King, D. L., \& Hunstead, R. W. 1997, ApJ, 486, 665

Puget, J.-L., Abergel, A., Bernard, J.-P., et al. 1996, A\&A, 308, L5+

Shioya, Y., Taniguchi, Y., Murayama, T., et al. 2002, ApJ, 576, 36

Songaila, A. \& Cowie, L. L. 2002, AJ, 123, 2183

Spergel, D. N., Verde, L., Peiris, H. V., et al. 2003, ApJS, 148, 175

VandenBerg, D. A., Swenson, F. J., Rogers, F. J., Iglesias, C. A., \& Alexander, D. R. 2000, ApJ, 532, 430 